\title{
ARTIGOS DE REVISÃO
APRENDIZAGEM ORGANIZACIONAL, GESTÃO DO
CONHECIMENTO E CAPACIDADES DINÂMICAS:
PROPOSTA DE UM MODELO TEÓRICO RELACIONAL
}

\author{
Rodrigo Franklin Frogeri \\ Doutorando em Sistemas de Informação e Gestão do Conhecimento pela \\ Fundação Mineira de Educação e Cultura, Brasil. Professor do Centro \\ Universitário do Sul de Minas, Brasil. \\ E-mail: rodrigoff@fumec.edu.br \\ Silmar Vasconcelos \\ Mestre em Sistemas de Informação e Gestão do Conhecimento pela \\ Fundação Mineira de Educação e Cultura, Brasil. \\ E-mail: silmar vasconcelos@yahoo.com \\ Janaina Soares Silva Pereira França \\ Mestre em Sistemas de Informação e Gestão do Conhecimento pela \\ Fundação Mineira de Educação e Cultura, Brasil. \\ E-mail: janainafranca.bh@gmail.com \\ Daniel Jardim Pardini \\ Doutor em Administração pela Universidade Federal de Minas Gerais, \\ Brasil. Professor da Fundação Mineira de Educação e Cultura, Brasil. \\ E-mail: pardini@fumec.br

\section{Daniela Assis Alves Ferreira} \\ Doutoranda em Sistemas de Informação e Gestão do Conhecimento pela \\ Fundação Mineira de Educação e Cultura, Brasil. Professora do Centro \\ Universitário Metodista Izabela Hendrix, Brasil. \\ E-mail: daniassis@gmail.com
}

\begin{abstract}
Resumo
Diante da necessidade de novas configurações dos ambientes organizacionais, a aprendizagem organizacional passou a ser considerada um fator fundamental para a inovação, vantagem competitiva e a gestão do conhecimento. É nesse sentido que este estudo tem como objetivo apresentar um modelo relacional entre os temas aprendizagem organizacional, gestão do conhecimento e capacidades dinâmicas. Tal abordagem se justifica mediante a possibilidade de integração conceitual entre os temas, por se tratar de assuntos complementares quando analisados em um contexto mais amplo, despertando interesse dos autores em propor um modelo teórico. Para alcançar o propósito definido, foi realizada uma revisão da literatura sobre as temáticas centrais em busca de estudos que fornecessem suporte teórico para uma ótica integrativa entre os temas. Com base nas discussões apresentadas, foi desenvolvido um modelo teórico abrangente, conciso e alinhado à literatura. $O$ estudo evidenciou que a aprendizagem organizacional, bem como o seu processo precedente, mudança transformacional são fatores-chave para o início de uma atividade de inovação, auxiliando a institucionalização do conhecimento, que, dentro do modelo proposto, pode levar a um esquecimento organizacional como forma de adaptação aos novos processos e conhecimentos. A gestão do conhecimento institucionalizado é aplicada, ainda dentro da ideia de capacidades dinâmicas, como a atividade que direciona a inovação e o aumento do desempenho organizacional, concluindo a estrutura das capacidades dinâmicas.
\end{abstract}

Palavras-chave: Aprendizagem organizacional. Gestão do conhecimento. Capacidades dinâmicas. Esquecimento organizacional. Mudança Transformacional.

Perspectivas em Gestão \& Conhecimento, João Pessoa, v. 9, n. 2, p. 24-39, maio/ago. 2019.

DOI: http://dx.doi.org/10.21714/2236-417X2019v9n2p24

http://periodicos.ufpb.br/ojs2/index.php/pgc. ISSN: 2236-417X. Publicação sob Licença (cc) EY-NC-ND 


\title{
ORGANIZATIONAL LEARNING, KNOWLEDGE MANAGEMENT AND DYNAMIC CAPACITIES: PROPOSAL OF A RELATIONAL THEORETICAL MODEL
}

\begin{abstract}
Faced with the need for new configurations of organizational environments, organizational learning has become a key-factor for innovation, competitive advantage and knowledge management. Hence, this study aims to present a relational model between the themes organizational learning, knowledge management and dynamic capabilities. Such approach is justified by the possibility of conceptual integration between the themes, once they are complementary subjects when analyzed in a broader context, arousing the interest of the authors in proposing a theoretical model. To achieve the defined objective, a literature review was conducted on the central themes. We search studies that would provide theoretical support for an integrative view between the themes. Based on the discussions presented, we developed a comprehensive theoretical model aligned to the literature. The study demonstrated that organizational learning, as well as its preceding process, transformational change are key-factors for the initiation of an innovation activity. Those process assists the institutionalization of knowledge, which, within the proposed model, can lead to an organizational forgetfulness as a way to adapt to new processes and knowledge. The institutionalized knowledge management is applied, still within the idea of dynamic capabilities, as the activity that drives innovation and increased organizational performance, concluding the structure of dynamic capabilities.
\end{abstract}

Keywords: Organizational learning. Knowledge management. Dynamic Capabilities. Organizational forgetfulness. Transformational change.

\section{INTRODUÇÃO}

No final da década de 1980 a mudança do paradigma industrial para o novo arcabouço centrado na informação levou as organizações a enfrentarem condições de incertezas, ambientes de intensa transformação e competição acirrada. Esses fatores direcionaram as organizações para uma modificação generalizada no seu foco produtivo. Iniciava-se a era da informação, um período em que o ambiente fabril dava lugar a um mundo de ideias e conhecimentos (MARANALDO, 1989; CORRÊA; RIBEIRO; KERR PINHEIRO, 2017). Neste contexto histórico de mudança, o conhecimento organizacional passou a ter um valor expressivo para os negócios, tendo a sua base conceitual estabelecida em uma série de trabalhos elaborados por Hayek (1945) e Penrose (2006), estudos estes voltados para a área econômica, e trabalhos filosóficos desenvolvidos por Dewey e Bentley (1948), Dewey (1998) e Polanyi (1966); mas somente no fim dos anos 1980 que o tema conhecimento organizacional ganhou destaque com os trabalhos de Nonaka (1991) e Nonaka e Takeuchi (1997) (BRĂTIANU, 2018; CHERMAN; ROCHA-PINTO, 2013).

Muito além das escolas clássicas da administração que iniciaram seus postulados científicos com Henry Ford, Henri Fayol e Frederick Taylor, com princípios mecanicistas que representavam a desumanização do trabalho industrial, chegara a hora de estudar novas metodologias que pudessem extrair não só a capacidade laboral, mas principalmente a capacidade intelectual dos funcionários.

A competitividade japonesa iniciada nos anos 1970 obrigou muitas empresas americanas, e posteriormente as europeias, a reagirem diante do inovador desenho que despontava no mercado. Ao final dos anos 1980 emergiam os primeiros estudos científicos direcionados a mecanismos de gestão que elucidavam para as empresas a necessidade de aprendizado constante para conviver com a cultura empreendedora e com o risco das inovações (OUCHI, 1985).

A Aprendizagem Organizacional (AO) tornou-se uma área emergente nos campos organizacionais e científicos na década de 1990, sobretudo após a publicação dos estudos de

Perspectivas em Gestão \& Conhecimento, João Pessoa, v. 9, n. 2, p. 24-39, maio/ago. 2019. 
Davenport e Prusak (1998). Senge (1990), em seus trabalhos sobre aprendizagem organizacional, comenta que o ser humano vem ao mundo motivado a aprender, explorar e experimentar, mas a maioria das instituições sociais são orientadas para controlar o indivíduo, ao invés de lhes proporcionar condições de aprendizagem.

Diante da necessidade de mudança e novas configurações dos ambientes organizacionais, a AO passou a ser considerada um fator fundamental para a inovação, vantagem competitiva e a gestão do conhecimento (MENDES, 2003; ŠKERLAVAJ, SONG; LEE, 2010). A capacidade de aprender torna-se fator preditivo à criação do conhecimento (SANZ-VALLE et al., 2011; SUTANTO, 2017), o que direciona a aprendizagem organizacional e a gestão do conhecimento como parte de um processo que visa suportar o desempenho organizacional e as mudanças do ambiente, processo este denominado pela literatura de capacidades dinâmicas (ZOLLO; WINTER, 2002; TEECE, 2007). As características dos temas possibilitam integrações conceituais, despertando interesse dos autores deste estudo em buscar um modelo relacional entre aprendizagem organizacional, gestão do conhecimento e capacidades dinâmicas, por considerar que são enfoques correlacionados que se complementam em um contexto mais amplo. É importante ressaltar a contribuição deste estudo para a comunidade científica, por possibilitar que diferentes linhas de pesquisa sejam abordadas em um estudo único, promovendo inferências interdisciplinares.

$\mathrm{O}$ estudo teve como objetivo apresentar um modelo relacional entre os temas aprendizagem organizacional, gestão do conhecimento e capacidades dinâmicas, apoiando-se em uma revisão da literatura sobre as temáticas centrais em busca de estudos que fornecessem suporte teórico para uma ótica integrativa entre os temas.

Além desta introdução, o estudo está estruturado em cinco tópicos: (i) Aprendizagem Organizacional; (ii) Gestão do Conhecimento e Capacidades Dinâmicas; (iii) Aprendizagem Organizacional e Gestão do Conhecimento versus Capacidades Dinâmicas; (iv) Proposta de um Modelo Relacional e (v) Conclusão. A primeira parte do artigo conceitua aprendizagem organizacional e apresenta as suas principais características. Na sequência, os temas gestão do conhecimento e capacidades dinâmicas são contextualizados, sendo relacionados à aprendizagem organizacional. No terceiro tópico, apresenta-se o aporte teórico para a proposta de um modelo relacional entre os temas. O último tópico apresenta a conclusão do trabalho, proposta de trabalhos futuros e limitações.

\section{REFERENCIAL TEÓRICO}

\subsection{APRENDIZAGEM ORGANIZACIONAL}

Os primeiros estudos em Aprendizagem Organizacional (AO) remetem à década de 1950, porém, somente a partir dos anos 1990 que o tema se desenvolveu, quando é abordada a aprendizagem individual versus organizacional, a aprendizagem de ciclo simples (mudanças incrementais de pequeno impacto) versus de ciclo duplo (mudança nos valores organizacionais/cultura organizacional) e a cognição versus comportamento (TAKAHASHI; FISCHER, 2008; ANTONELLO; GODOY, 2009). Alguns anos depois, a AO passa a ser vista como fundamental para a competitividade das empresas e como fator de adaptação às mudanças constantes do ambiente. A AO destaca-se como um meio de aplicar a gestão do conhecimento pela necessária interação entre pessoas, o que relaciona o tema às áreas da inovação e efetivamente à gestão do conhecimento (MENDES, 2003; ŠKERLAVAJ; SONG; LEE, 2010; SANTANA; SOUZA-SILVA, 2012; ALMEIDA; SILVA, 2016).

Desde então, a criação do conhecimento é vista como um fator intrínseco à capacidade de aprendizagem, que por sua vez estimula a inovação organizacional e exige um alto nível de aprendizagem organizacional (SANZ-VALLE et al., 2011; SUTANTO, 2017). Nonaka e Takeuchi

Perspectivas em Gestão \& Conhecimento, João Pessoa, v. 9, n. 2, p. 24-39, maio/ago. 2019. 
(1997) propõem que as interações entre os indivíduos e os grupos dentro de uma organização são a fonte do conhecimento organizacional, que por meio de um processo de compartilhamento, criação, justificação, construção e difusão é gerado o conhecimento. Nonaka, Toyama e Hirata (2011) evoluem o modelo de criação do conhecimento de Nonaka e Takeushi (1997), atribuindo-Ihe o fator cultural, por considerar que é um processo relacionado às crenças e à cultura do ambiente, definindo o conhecimento como um fruto das interações e do feedback entre pessoas. Nessa perspectiva, Camillis e Antonello (2010) definem a aprendizagem como algo que emerge das interações sociais, podendo-se inferir um processo evolutivo da aprendizagem ao conhecimento. Para Scatolin (2015), a organização por si mesma não pode criar conhecimento sem a iniciativa do indivíduo e a interação que ocorre dentro do grupo.

Tendo como base a suposição de que para responder à mudança, a organização tem que aprender continuamente (ANTONELLO; GODOY, 2009), as abordagens dadas pela academia em AO divergem quanto a área de enfoque, enquanto alguns pesquisadores direcionam os esforços para a gestão estratégica e gestão do conhecimento (CHIVA; ALEGRE, 2005; D'ARISBO et al., 2016; ABEL, 2015), outros buscam na gestão de pessoas e na psicologia (SCORSOLINI-COMIN, INOCENTE; MIURA, 2011) explicar como se dá a aprendizagem organizacional. Contudo, independentemente do campo investigado, o tema tem seu aporte na mudança organizacional ou, como Takahashi e Fisher (2008) caracterizam, nos processos de mudança transformacional, aqueles capazes de alterar a organização como um todo (COUTODE-SOUZA; TOMEI, 2008).

Para Couto-De-Souza e Tomei (2008) e Barreto et al. (2013), o sucesso da mudança nas organizações está vinculado à liderança transformacional, caracterizada pelo engajamento dos indivíduos em busca de laços que aumentem o nível de motivação de líderes e liderados. Jung, Chow e Wu (2003) consideram que a liderança transformacional está ligada diretamente à inovação organizacional, caracterizando o líder transformacional como sendo dotado de quatro únicas, mas inter-relacionadas competências: (i) motivação inspiradora (articular uma atraente e/ou sugestiva visão); (ii) estimulação intelectual (promoção da criatividade e inovação); (iii) influência idealizada (papel carismático), e considerações individualizadas (coaching e mentoria). Os achados de Jung, Chow e Wu (2003) ratificam a Samad (2012), que observou a liderança transformacional e a inovação fatores com influência significativa para o desempenho organizacional, podendo-se estabelecer uma estreita relação entre elementos que compõem a aprendizagem organizacional e o próprio desempenho das organizações.

Patriotta (2003), ao observar a dinâmica do processo evolutivo de aprendizagem ao conhecimento, enfatiza que a institucionalização do conhecimento é condicional ao desempenho organizacional, criando assim um sistema classificatório de conhecimento nas organizações. A partir do modelo de Patriotta (2003), é possível vincular os estudos em "aprendizagem organizacional ao tipo e grau de profundidade da mudança e ao movimento do conhecimento organizacional (da criação à institucionalização do conhecimento)" (TAKAHASHI; FISCHER, 2008, p. 6).

Os estudos de Patriotta (2003) apoiaram Takahashi e Fisher (2008) a definirem os elementos conceituais que justificam a relação entre competências e aprendizagem, além de abranger o processo de mudança e do fluxo de conhecimento. Destarte, foi proposto por Takahashi e Fisher (2008) que a aprendizagem organizacional envolva quatro elementos, a saber: i) inicia-se com um processo de mudança transformacional; ii) envolve dinamicamente os vários níveis (indivíduos, grupos e organização) pelo qual se dá a criação, utilização e institucionalização do conhecimento; iii) opera no âmbito coletivo, abrangendo aspectos cognitivos, comportamentais e culturais; iv) seu resultado, ou conteúdo, é o próprio conhecimento, fonte e resultado de seu background (história, hábitos e experiências), 
expresso no desenvolvimento das competências organizacionais (aquisição, alavancagem ou manutenção) (TAKAHASHI; FISCHER, 2008).

Assim como há o processo de criação, utilização e institucionalização do conhecimento, Remor et al. (2010) abordam a importância do esquecimento organizacional, ao considerarem que este interfere no processo da aprendizagem. Holan e Philips (2005) afirmam que o conhecimento organizacional está embutido em algum tipo de memória organizacional, que não desaparece apesar de indivíduos irem e virem.

Holan e Philips (2005) destacam ainda que o esquecimento organizacional possui pelo menos três contextos: o primeiro estabelece que, simplesmente ser capaz de criar ou transferir conhecimento não é suficiente e que evitar o esquecimento do conhecimento recém-adquirido é uma parte importante da aprendizagem eficaz. O segundo contexto denota que a memória organizacional decai ao longo do tempo e partes importantes do conhecimento podem ser esquecidas se a memória organizacional não for mantida, ou retroalimentada. Em um terceiro contexto, estabelece-se que o esquecimento é, às vezes, uma necessidade organizacional, que revela associação às reconfigurações dos ambientes, a novos processos e tecnologias que precisam de espaço e sobreposição do antigo para se institucionalizarem, caso contrário, a incapacidade de esquecer ou não perceber as mudanças pode levar ao fracasso. Holan e Philips (2005) concluem que tanto evitar o esquecimento organizacional quanto maximizar a perda do conhecimento são importantes para a organização. Holan e Philips (2005, p. 1611), baseiam-se nos pressupostos de que "se o conhecimento crítico foi esquecido, então a competitividade foi perdida e o esquecimento deveria ter sido evitado. Se o conhecimento esquecido era "ruim" ou estava interferindo ativamente na aplicação de um conhecimento mais apropriado, o esquecimento foi uma ocorrência positiva". Nesse contexto, reflete-se que uma nova atividade pode ser incorporada ao processo de AO; a identificação e classificação do conhecimento para esquecimento.

Em um contexto geral, tanto a criação quanto o esquecimento do conhecimento fazem parte do processo de aprendizagem organizacional e da gestão do conhecimento que, conforme observado por Eisenhardt e Martin (2000) e Giniuniene e Jurksiene (2015), evidencia e incorpora as capacidades dinâmicas aos processos internos das empresas.

\subsection{GESTÃO DO CONHECIMENTO E CAPACIDADES DINÂMICAS}

O desenvolvimento das tecnologias da informação e comunicação ampliou o dinamismo na troca de informações dos mercados e agilidade nos negócios, aumentando a competitividade e obrigando as empresas a se adaptarem rapidamente às mudanças do ambiente (COSTA; PORTO; GONÇALVES, 2013). Para Drucker (1988, p. 12) "a fonte básica de toda a vantagem competitiva está na capacidade relativa da empresa de aprender mais rápido do que seus concorrentes", necessitando que as organizações migrem da era da competição baseada em recursos para uma nova era baseada em conhecimento (SENGE, 1995). Nonaka (1991, p. 27) reflete sobre a vantagem competitiva nesse cenário e considera que "numa economia onde a única certeza é a incerteza, apenas o conhecimento é fonte segura de vantagem competitiva". Em um contexto de economia baseada no conhecimento, o ser humano passa a ser o ativo mais importante da organização, e a Gestão do Conhecimento (GC) uma condição necessária para a obtenção de êxito e sucesso nas empresas (NEVES; CERDEIRA, 2018; PEREIRA; SILVA, 2018).

A GC pode ser compreendida como "um processo sistemático de identificação, criação, renovação e aplicação dos conhecimentos que são estratégicos na vida de uma organização, é a administração dos ativos de conhecimento" (NONAKA; TAKEUCHI, 1997, p. 64), que modifica políticas, processos, atividades, tecnologias, ferramentas, e a própria cultura da organização (ESCRIVÃO; NAGANO; ESCRIVÃO FILHO, 2011; VALIO; GONZALEZ; MARTINS, 2015).

Perspectivas em Gestão \& Conhecimento, João Pessoa, v. 9, n. 2, p. 24-39, maio/ago. 2019. 
A GC trata da criação do conhecimento organizacional como uma interação contínua e dinâmica entre o conhecimento tácito (pessoal, específico ao contexto) e o conhecimento explícito (transmissível em linguagem formal e sistemática) (ESCRIVÃO; NAGANO; ESCRIVÃO FILHO, 2011; NONAKA; TAKEUCHI, 1997). A criação do conhecimento organizacional é um processo em espiral, que começa no nível individual e vai subindo, ampliando comunidades de interação que cruzam fronteiras entre seções, departamentos, divisões e organizações (SCATOLIN, 2015).

Para Nonaka e Takeuchi (1997), o processo de conversão do conhecimento (espiral do conhecimento) inicia por meio da socialização, em que trocas de experiências, observação e treinamentos possibilitam o compartilhamento de conhecimentos tácitos. A próxima etapa é a externalização, onde a conversão do conhecimento tácito compartilhado deve ser explicitada, apresentado ao grupo. A etapa de combinação trata da padronização do conhecimento explícito, transformando-o em manuais, documentos e/ou regras. Por fim, o conhecimento explicitado passa a ser utilizado e disseminado pela organização, há a sua internalização por parte das pessoas, iniciando assim um novo ciclo da espiral (ESCRIVÃO; NAGANO; ESCRIVÃO FILHO, 2011; NONAKA; TAKEUCHI, 1997).

A troca de conhecimentos internalizados e a sua gestão adequada contribui para a geração de ideias, novos produtos e aumento da produtividade nas organizações (VALDEZJUÁREZ; LEMA; MALDONADO-GUZMÁN, 2016). Nesse sentido, as organizações constroem, reconfiguram e integram suas competências organizacionais e modelos de aprendizado em busca de identificar a necessidade ou oportunidade de mudança (COSTA; PORTO; GONÇALVES, 2013; VALDEZ-JUÁREZ; LEMA; MALDONADO-GUZMÁN, 2016).

Diante de um cenário dinâmico, com sistemáticas mudanças tecnológicas, o tema capacidades dinâmicas ganha relevância na literatura buscando identificar o modo como as organizações podem sustentar uma vantagem competitiva, adaptando-se aos ecossistemas empresarias por meio da inovação e da colaboração com outras empresas, entidades e instituições (HELFAT; PETERAF, 2009; TEECE, 2007). Para Valdez-Juárez, Lema e MaldonadoGuzmán (2016, p. 147), a GC, relacionada às capacidades dinâmicas, "serve para explicar o sucesso ou o fracasso, a geração de resultados financeiros, vantagem competitiva e inovação ao longo do tempo de uma organização".

Alguns autores associam o desenvolvimento das capacidades dinâmicas ao próprio dinamismo do ambiente (TEECE; PISANO; SCHUEN, 1997; WANG; AHMED, 2007), enquanto outros, como Zollo e Winter (2002, p. 4) defendem que "há empresas que desempenham o conjunto de requisitos de integrar, construir e reconfigurar suas competências mesmo em ambientes pouco dinâmicos e com baixas taxas de mudanças".

Winter (2003) e Andreeva e Chaika (2006) consideram que as capacidades dinâmicas estão fundamentadas na existência de mecanismos rotineiros, que permitem a reconfiguração das capacidades das empresas; alinhando-se ao pensamento de Eisenhardt e Martin (2000), que consideram as capacidades dinâmicas como as rotinas organizacionais e estratégicas pelas quais as empresas conseguem novas configurações de recursos à medida que os mercados emergem, colidem, se dividem, evoluem e morrem. Teece (2007) complementa os aspectos conceituais do tema apresentando as capacidades dinâmicas como uma forma de criar e proteger os ativos intangíveis que suportam o desempenho das organizações a longo prazo.

Para relacionar a definição de capacidades dinâmicas aos seus elementos componentes, Meirelles e Camargo (2012) elaboraram o Quadro 1, destacando-se o aprendizado nas suas linhas teóricas. 
Quadro 1 - Capacidades dinâmicas e elementos componentes

\begin{tabular}{|l|l|l|}
\hline \multicolumn{1}{|c|}{ Autores } & \multicolumn{1}{|c|}{ Capacidades Dinâmicas } & \multicolumn{1}{c|}{ Elementos Componentes } \\
\hline $\begin{array}{l}\text { Zollo e Winter } \\
\text { (2002) }\end{array}$ & $\begin{array}{l}\text { Padrão aprendido e estável de } \\
\text { atividade coletiva por meio do qual a } \\
\text { organização, sistematicamente, gera } \\
\text { e modifica suas rotinas operacionais } \\
\text { em busca de melhoria na } \\
\text { efetividade. }\end{array}$ & $\begin{array}{l}\text { - Processos de acumulação de } \\
\text { experiências; articulação de conhecimento } \\
\text { e codificação de conhecimento; } \\
\text { - Ciclo recursivo de aprendizagem. }\end{array}$ \\
\hline Winter (2003) & $\begin{array}{l}\text { Capacidades para operar, estender, } \\
\text { modificar ou criar capacidades } \\
\text { comuns. }\end{array}$ & $\begin{array}{l}\text { - Existência de uma coleção de rotinas de } \\
\text { alto nível que definem padrões de } \\
\text { atividades; } \\
\text { - Comprometimento de longo prazo de } \\
\text { recursos especializados; }\end{array}$ \\
& $\begin{array}{l}\text { - Exercício de uso das capacidades } \\
\text { dinâmicas; } \\
\text { - Investimentos em aprendizagem. }\end{array}$ \\
\hline Bygdas (2006) & $\begin{array}{l}\text { Processos e estruturas ativas, } \\
\text { redes fragmentadas de } \\
\text { procedimentos e entendimentos } \\
\text { soltos que desenvolvem práticas } \\
\text { mais eficientes que não são } \\
\text { facilmente imitáveis. }\end{array}$ & $\begin{array}{l}\text { - Conhecimento prévio significativo; } \\
\text { - Unidades de processamento de } \\
\text { conhecimento; } \\
\text { - Redes de conexão das unidades de } \\
\text { processos; } \\
\text { - Estruturas de conhecimento e; processos } \\
\text { de ativação. }\end{array}$ \\
\hline
\end{tabular}

Fonte: Adaptado de Meirelles e Camargo (2014)

Os estudos de Meirelles e Camargo (2014) ampliam os achados de Andreeva e Chaika (2006), apontando alguns fatores no qual as capacidades dinâmicas dependem, como a existência das habilidades de liderança e a capacidade de mudança organizacional, sendo esta última considerada central e com a definição de pontos chave, como: desenvolvimento de habilidades não específicas; desenvolvimento de lealdade das pessoas às mudanças; e criação de mecanismos organizacionais relevantes.

Em uma perspectiva relacional, os elementos componentes do Quadro 1 denotam uma estreita associação entre aprendizagem (ciclo recursivo de aprendizagem; investimentos em aprendizagem; acumulação de experiências), gestão do conhecimento (articulação de conhecimento e codificação de conhecimento; unidades de processamento de conhecimento; estruturas de conhecimento; coleção de rotinas de alto nível que definem padrões de atividades) e os conceitos de capacidades dinâmicas (padrão aprendido e estável de atividade coletivas; capacidades para operar, estender, modificar ou criar capacidades comuns; desenvolvem práticas mais eficientes que não são facilmente imitáveis).

Nessa perspectiva, associar os elementos que envolvem o tema capacidades dinâmicas aos princípios de aprendizagem organizacional e gestão do conhecimento, torna-se um fator relevante para a literatura como uma forma de integração conceitual, sendo abordado a seguir.

\subsection{APRENDIZAGEM ORGANIZACIONAL E GESTÃO DO CONHECIMENTO VERSUS CAPACIDADES DINÂMICAS}

A temática aprendizagem organizacional foi foco dos primeiros estudos na década de 1950 em que se buscava a compreensão do sucesso das organizações (GRINSVEN; VISSER, 2011). No entanto, a competitividade atual e a necessidade de inovação em mercados 
dinâmicos levaram os pesquisadores a buscar novas configurações desta temática (COPE, 2003; WANG; CHUGH, 2014).

Grinsven e Visser (2011) destacam dois assuntos mais influentes na literatura da aprendizagem organizacional, sendo o primeiro empoderamento, que se relaciona ao grau de descentralização das responsabilidades de decisão nas organizações (CHIVA; ALEGRE, 2005) e o segundo, à conversão do conhecimento, cerne da gestão do conhecimento, referindo-se ao grau em que o conhecimento está sendo tácita e explicitamente disseminado nas organizações (NONAKA; TAKEUCHI, 1997; NONAKA; TOYAMA, 2003). Isto posto, Giniuniene e Jurksiene (2015), em uma abordagem voltada para a gestão do conhecimento, conceituam a aprendizagem organizacional como o processo operacional de obtenção de informação e de conversão em conhecimento. Çinar e Eren (2015) contribuem para a definição apresentando as atividades de aprendizagem organizacional como um processo dinâmico de avaliação do conhecimento organizacional, disseminação e criação.

Eisenhardt e Martin (2000), sugerem que o processo de aprendizagem das organizações gera novos conhecimentos, colocando em evidência as capacidades dinâmicas das empresas; enquanto que Giniuniene e Jurksiene (2015) consideram que a aprendizagem organizacional pode ser tratada como a forma de incorporar capacidades dinâmicas nos processos internos da empresa, ajudando a mediar a relação capacidades dinâmicas e 0 desempenho da empresa (GINIUNIENE; JURKSIENE, 2015), o que para Shane e Venkataraman (2000) tornam as capacidades dinâmicas uma fonte primária de vantagem competitiva.

Pereira, Cunha e Bulgacov (2013) exploram os vínculos do conhecimento, tanto com a gestão estratégica quanto com a vantagem competitiva, reconhecendo o conhecimento enquanto suporte para a geração das capacidades dinâmicas. A prática da gestão do conhecimento e aprendizagem organizacional contribuem de forma considerável na rotina da organização, mas estas podem não ser as únicas nem as principais funcionalidades percebidas na prática, mas como fatores que contribuem para o conseguimento das capacidades dinâmicas (PEREIRA; CUNHA; BULGACOV, 2013; ZANGISKI; LIMA; COSTA, 2009).

\section{PROPOSTA DE UM MODELO TEÓRICO RELACIONAL}

Seguindo o referencial teórico desta pesquisa, buscou-se estabelecer um modelo relacional dos conceitos de aprendizagem organizacional, gestão do conhecimento e capacidades dinâmicas. Assim, a aprendizagem organizacional foi observada como um processo de competências e aprendizagem (TAKAHASHI; FISHER, 2008), enquanto o tema Gestão do Conhecimento tem sua base nos trabalhos de Nonaka e Takeuchi (1997) e Nonaka, Toyama e Hirata (2011), já as capacidades dinâmicas são observadas sob diferentes pontos de vista, devido a sua abrangência conceitual. A seguir, o modelo proposto é apresentado na Figura 1.

O modelo tem o seu início por meio de uma mudança transformacional no ambiente, que implica em grandes alterações nos processos organizacionais, sendo um líder transformacional considerado o estilo ideal para a gestão desse tipo de mudança (JUNG; CHOW; WU, 2003; COUTO-DE-SOUZA; TOMEI, 2008; BARRETO et al., 2013). Jung, Chow e Wu (2003) e Takahashi e Fisher (2003) consideram a mudança transformacional o processo inicial para a inovação e aumento do desempenho organizacional (SAMAD, 2012). A mudança alimenta o processo de aprendizagem organizacional, conforme identificado por Takahashi e Fisher (2008). 
Figura 1 - Modelo relacional entre Aprendizagem Organizacional, Gestão do Conhecimento e capacidades dinâmicas

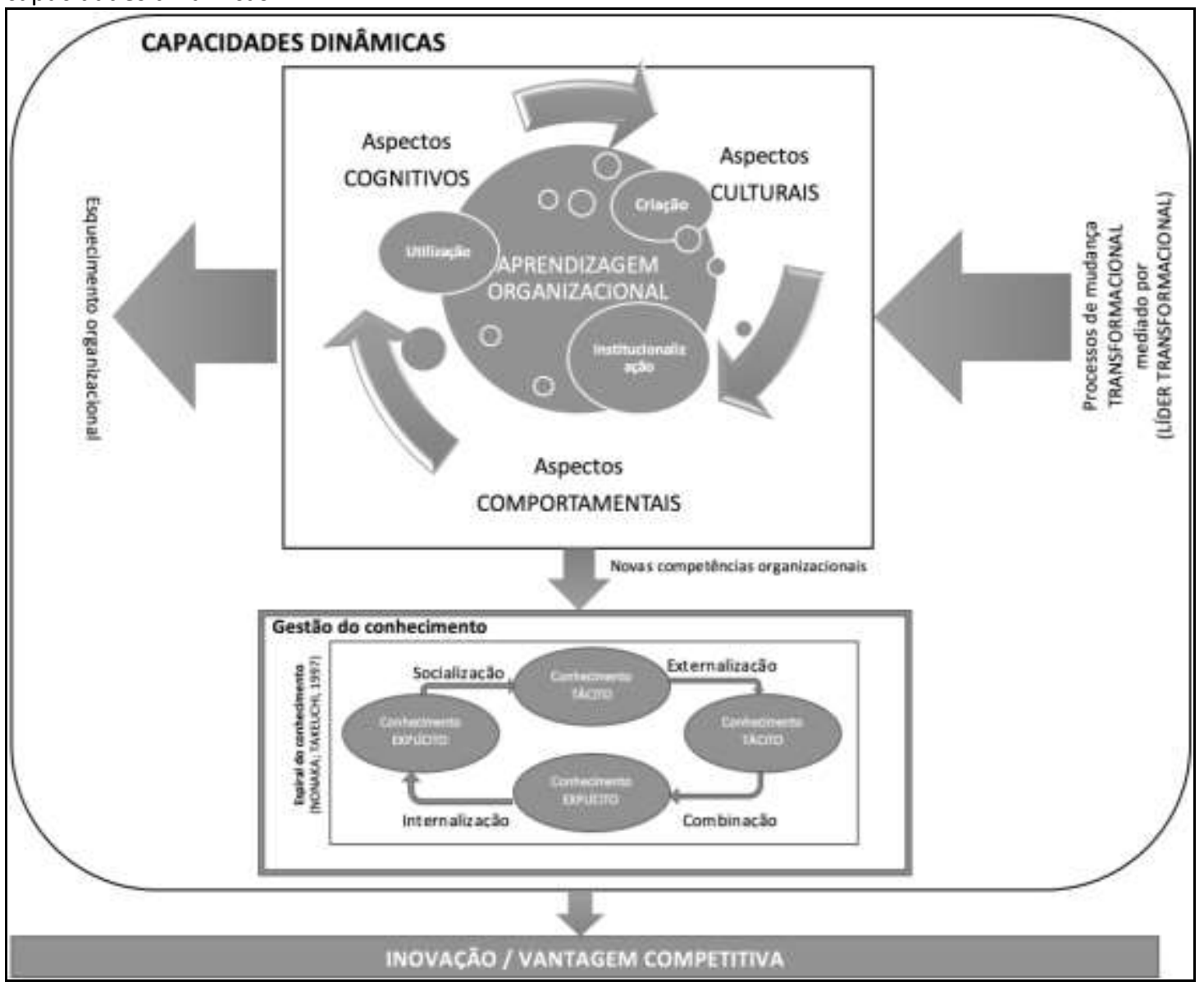

Fonte: Desenvolvida pelos autores (2019)

Os aspectos culturais, cognitivos e comportamentais atuam como fatores de resistência à criação, utilização e institucionalização do conhecimento (TAKAHASHI; FISHER, 2008). O conhecimento institucionalizado por meio da aprendizagem organizacional estabelece as novas competências organizacionais, que serão submetidas à espiral da gestão do conhecimento e sua abordagem sistêmica (NONAKA; TAKEUCHI, 1997; PEREIRA, CUNHA; BULGACOV, 2013). Considerou-se a GC envolta em um quadro pelo fato da espiral do conhecimento, por si só, não representar todas as condições para que ocorra a gestão do conhecimento (NONAKA; TAKEUCHI, 1997; NONAKA; TOYAMA, 2003).

Os novos conhecimentos suportam e colocam em evidência as capacidades dinâmicas das organizações (EISENHARDT; MARTIN, 2000), proporcionando a inovação e vantagem competitiva (GINIUNIENE; JURKSIENE, 2015; ÇINAR; EREN, 2015; SHANE; VENKATARAMAN, 2000). O processo central, aprendizagem organizacional, possui uma entrada e duas saídas, sendo a primeira saída, o próprio conhecimento ou nova competência, e a segunda saída o esquecimento organizacional, estabelecido como um fator dependente para que a aprendizagem organizacional gere o conhecimento institucionalizado (HOLAN; PHILIPS, 2004; REMOR et al., 2010).

Buscando estabelecer uma relação comparativa a outros modelos da literatura que abordaram assuntos correlatos, a seguir é apresentado o modelo teórico de Giniuniene e Jurksiene (2015) - Figura 2. Giniuniene e Jurksiene (2015) objetivaram estabelecer uma estrutura de associações entre capacidades dinâmicas, aprendizagem organizacional e inovação, além do impacto dessas associações no desempenho da empresa. 
Figura 2 - Modelo teórico proposto por Giniuniene e Jurksiene (2015)

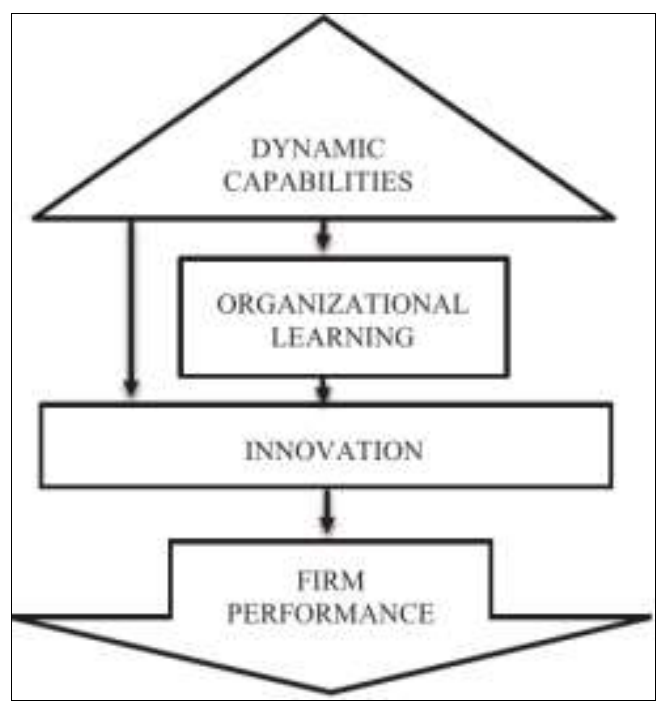

Fonte: Giniuniene e Jurksiene (2015, p. 4)

Observa-se no trabalho de Giniuniene e Jurksiene (2015) uma relação semelhante à apresentada neste estudo, contudo, sem um detalhamento do processo que pode ser considerado central no modelo, a aprendizagem organizacional, bem como os seus processos de entrada (mudança transformacional), saída (gestão do conhecimento e esquecimento organizacional), e os fatores de resistência (aspectos cognitivos, culturais e comportamentais) para a institucionalização do conhecimento na organização. A análise denota que o modelo proposto neste estudo tem uma correlação conceitual mais detalhada e um aporte teórico mais aprofundado se comparado ao estudo de Giniuniene e Jurksiene (2015).

\section{CONSIDERAÇÕES FINAIS}

O objetivo deste estudo foi apresentar um modelo relacional entre os temas aprendizagem organizacional, gestão do conhecimento e capacidades dinâmicas. Observou-se pela revisão da literatura que o tema capacidades dinâmicas tem sido considerado no meio acadêmico como bastante amplo e divergente no estabelecimento de uma definição única.

Destarte, este trabalho buscou nos processos dependentes das capacidades dinâmicas relacionar os conceitos que abrangem o assunto. A aprendizagem organizacional, bem como $o$ seu processo precedente, mudança transformacional, foram observados como fatores-chave para o início de uma atividade de inovação, auxiliando a institucionalização do conhecimento, que, dentro do modelo proposto, pode levar a um esquecimento organizacional como forma de adaptação aos novos processos e conhecimentos. Reflete-se que um processo de identificação e classificação do conhecimento pode ocorrer entre o contexto de $A O$ e $O$ esquecimento organizacional, a fim de que conhecimentos críticos não sejam descartados. A gestão do conhecimento institucionalizado é aplicada, ainda dentro da ideia de capacidades dinâmicas, como a atividade que direciona a inovação e o aumento do desempenho organizacional, concluindo a estrutura das capacidades dinâmicas. Todo o processo pode ser repetido a partir de uma mudança transformacional na organização.

Podemos considerar que o estudo traz implicações para o meio acadêmico e organizacional. No plano acadêmico permitiu que conceitos distintos na literatura pudessem ser observados de forma inter-relacionada, ampliando a possibilidade de abordagens interdisciplinares. O modelo desenvolvido, quando comparado a outro modelo semelhante,

Perspectivas em Gestão \& Conhecimento, João Pessoa, v. 9, n. 2, p. 24-39, maio/ago. 2019. 
apresentou-se com maior detalhamento dos processos internos e maior apropriação dos conceitos abordados. No plano organizacional, o estudo pode contribuir para uma ótica mais ampla das práticas que envolvem aprendizagem organizacional e gestão do conhecimento. 0 papel de destaque de um líder transformacional no início do processo pode delimitar perfis sob um plano estratégico organizacional que envolva as práticas discutidas. Ademais, a observação dos fatores de resistência à institucionalização do conhecimento como os aspectos culturais, cognitivos e comportamentais podem alertar gestores e líderes na adoção de práticas mitigadoras; ainda sob o aspecto organizacional, destacou-se a importância de que práticas antigas e sem valor para a organização sejam descartadas para que as novas práticas possam ser institucionalizadas.

Este estudo exige maiores aprofundamentos nas discussões a respeito do processo de classificação e identificação do conhecimento no contexto da aprendizagem organizacional. A observação das relações apresentadas pode ser explorada numa ótica reducionista ou de forma holística como causa da inovação e vantagem competitiva. A relação inovação e vantagem competitiva como saída de todo o processo também pode ser objeto de estudos futuros.

\section{REFERÊNCIAS}

ABEL, M. H. Knowledge map-based web platform to facilitate organizational learning return of experiences. Computers in Human Behavior, v. 51, p. 960-966, 2015. Disponível em: https://doi.org/10.1016/j.chb.2014.10.012. Acesso em: 15 ago. 2018.

ALMEIDA, N. C. P. DE; SILVA, J. C. DE S. Aprendizagem Organizacional e formação de gestores: como aprendem os gestores em uma indústria do setor petroquímico. REGE - Revista de Gestão, v. 22, n. 3, p. 381-402, 2016. Disponível em: http://www.revistas.usp.br/rege/article/view/111482. Acesso em: 15 ago. 2018.

ANDREEVA, T.; CHAIKA, V. Dynamic capabilities: what they need to be dynamic? Discussion Paper \#10(E)-2006. Institute of Management, St. Petersburg State University: SPb., 2006. Disponível em: https://dspace.spbu.ru/bitstream/11701/846/1/10\%28E\%29_2006.pdf. Acesso em: 15 ago. 2018.

ANTONELLO, C. S.; GODOY, A. S. Uma agenda brasileira para os estudos em aprendizagem organizacional. RAE - Revista de Administração de Empresas, v. 49, n. 3, p. 266-281, jul./set. 2009. Disponível em: http://dx.doi.org/10.1590/S0034-75902009000300003. Acesso em: 15 ago. 2018.

BARRETO, L. M. T. da S. et al. Cultura organizacional e liderança: uma relação possível? Revista de Administração, São Paulo, v. 48, n. 1, p. 34-52, mar. 2013. Disponível em: http://dx.doi.org/10.5700/rausp1072. Acesso em: 15 ago. 2018.

BYGDAS, A. L. Enacting dynamic capabilities in distributed organisational environments. Proceedings of the EGOS Conference, Bergen, Norway, 22. jul, 2006.

BRĂTIANU, C. A holistic view of the organizational knowledge dynamics. HOLISTICA - Journal of Business and Public Administration, v. 9, n. 2, p. 7-22, 2018. 
CAMILLIS, P. K.; ANTONELLO, C. S. Um estudo sobre os processos de aprendizagem dos trabalhadores que não exercem função gerencial. RAM-Revista de Administração Mackenzie, São Paulo, v. 11, n. 2, p. 4-42, abr. 2010. Disponível em: http://dx.doi.org/10.1590/S167869712010000200002. Acesso em: 15 ago. 2018.

CHERMAN, A.; ROCHA-PINTO, S. R. Gestão do conhecimento no Brasil: visão da academia. Revista Pensamento Contemporâneo em Administração, v. 7, n. 1, p. 92-107, 2013. Disponível em: http://www.spell.org.br/documentos/ver/30831/gestao-do-conhecimento-nobrasil--visao-da-academia/i/pt-br. Acesso em: 15 ago. 2018.

CHIVA, R.; ALEGRE, J. Organizational Learning and Organizational Knowledge: Towards the Integration of Two Approaches. Management Learning, v. 36, n. 1, p. 49-68, mar. 2005. Disponível em: https://doi.org/10.1177/1350507605049906. Acesso em: 15 ago. 2018.

ÇINAR, F.; EREN, E. Organizational learning capacity impact on sustainable innovation: the case of public hospitals. Procedia - Social and Behavioral Sciences, v. 181, p. 251-260, maio 2015. Disponível em: https://doi.org/10.1016/j.sbspro.2015.04.886. Acesso em: 15 ago. 2018.

COPE, J. Entrepreneurial learning and critical reflection. Management Learning, v. 34, n. 4, p. 429-450, dez. 2003. Disponível em: https://doi.org/10.1177/1350507603039067. Acesso em: 15 ago. 2018.

CORRÊA, F.; RIBEIRO, J. S. DE A. N.; KERR PINHEIRO, M. M. Aspectos da economia da informação: arquétipo conceitual, econômico e social. Inf. Inf., v. 22, n. 1, p. 185-214, 2017. Disponível em: http://dx.doi.org/10.5433/1981-8920.2017v22n1p185. Acesso em: 26 abr. 2019.

COSTA, P. R. da; PORTO, G. S.; GONÇALVES, M. N. Gestão global das capacidades dinâmicas de inovação e cooperação: um estudo multicaso com multinacionais brasileiras da indústria petroquímica. In: ENCONTRO DE ESTUDOS EM ESTRATÉGIA, 6., 2013, Bento Gonçalves/RS. Anais... Bento Gonçalves/RS: ANPAD, 2013. p. 1-16. Disponível em: http://www.anpad.org.br/admin/pdf/3Es315.pdf. Acesso em: 15 ago. 2018.

COUTO-DE-SOUZA, C. L.; TOMEI, P. A. Impactos de uma liderança transacional em um processo de mudança transformacional. Revista Psicologia: Organizações e Trabalho, Florianópolis, v. 8, n. 2, p. 98-120, jan. 2008.2 Disponível em: https://periodicos.ufsc.br/index.php/rpot/article/view/10161. Acesso em: 15 ago. 2018.

D'ARISBO, A. et al. Criação de conhecimento sob a ótica da aprendizagem organizacional em uma empresa criativa. Revista de Administração da UFSM, Santa Maria, v. 9, n. 1, p. 83-101, jan./mar. 2016. Disponível em: http://dx.doi.org/10.5902/1983465911271. Acesso em: 15 ago. 2018.

DAVENPORT, T. H.; PRUSAK, L. Conhecimento empresarial. Rio de Janeiro: Campus, 1998.

DEWEY, J. The essential dewey: pragmatism, education, democracy. v. 1. Indiana: Indiana University Press, 1998.

DEWEY, J.; BENTLEY, A. Knowing and the known. Disponível em: <www.aier.org>. Acesso em: 24 abr. 2017.

Perspectivas em Gestão \& Conhecimento, João Pessoa, v. 9, n. 2, p. 24-39, maio/ago. 2019. 
DRUCKER, P. The Coming of the new organization. Harvard Business Review, v. 66, n. 1, p. 4553, 1988.

EISENHARDT, K. M.; MARTIN, A. J. Dynamic capabilities: what are they? Strategic Management Journal, v. $21, \quad$ n. $10-11$, p. 1105-1121, out./nov. 2000. Disponível em: https://pdfs.semanticscholar.org/00c6/7a6c7c38a95cd639846dbf764936713de0d9.pdf. Acesso em: 15 ago. 2018.

ESCRIVÃO, G.; NAGANO, M. S.; ESCRIVÃO FILHO, E. A gestão do conhecimento na educação ambiental. Perspectivas em Ciência da Informação, Belo Horizonte, v. 16, n. 1, p. 92-110, jan./mar. 2011. Disponível em: http://dx.doi.org/10.1590/S1413-99362011000100006. Acesso em: 15 ago. 2018.

GINIUNIENE, J.; JURKSIENE, L. Dynamic capabilities, innovation and organizational learning: interrelations and impact on firm performance. Procedia - Social and Behavioral Sciences, v. 213, p. 985-991, dez. 2015. Disponível em: https://doi.org/10.1016/j.sbspro.2015.11.515. Acesso em: 15 ago. 2018.

GRINSVEN, M. V.; VISSER, M. Empowerment, knowledge conversion and dimensions of organizational learning. The Learning Organization, v. 18, n. 5, p. 378-391, 2011. Disponível em: https://doi.org/10.1108/09696471111151729. Acesso em: 15 ago. 2018.

HAYEK, F. A. The use of knowledge in society. The American Economic Review, v. 35, n. 4, p. 519-530, 1945. Disponível em: www.jstor.org/stable/1809376. Acesso em: 15 ago. 2018.

HELFAT, C. E.; PETERAF, M. A. Understanding dynamic capabilities: progress along a developmental path. Strategic Organization, v. 7, n. 1, p. 91-102, fev. 2009. Disponível em: < https://doi.org/10.1177/1476127008100133>. Acesso em: 15 ago. 2018.

HOLAN, P. M. DE; PHILLIPS, N. Remembrance of Things Past? The Dynamics of Organizational Forgetting. Management Science, v. 50, n. 11, p. 1603-1613, 2004.

JUNG, D. I.; CHOW, C.; WU, A. The role of transformational leadership in enhancing organizational innovation: hypotheses and some preliminary findings. Leadership Quarterly, v. 14, n. 4-5, p. 525-544, ago./out. 2003. Disponível em:

https://doi.org/10.1016/S1048-9843(03)00050-X. Acesso em: 15 ago. 2018.

MARANALDO, D. Estratégias para a competitividade. São Paulo: Produtividade, 1989.

MENDES, I. A. C. Aprendizagem organizacional e gestão do conhecimento: um desafio a ser enfrentado. Revista Latino-Americana de Enfermagem, Ribeirão Preto, v. 11, n. 3, p. 269-271, maio/jun. 2003. Disponível em: http://dx.doi.org/10.1590/S0104-11692003000300001. Acesso em: 15 ago. 2018.

MEIRELLES, D. S.; CAMARGO, Á. A. B. Capacidades dinâmicas: o que são e como identificá-las? RAC - Revista de Administração Contemporânea, Rio de Janeiro, v.18, Ed. Esp., p. 41-64, dez. 2014. Disponível em: 10.1590/1982-7849rac20141289. Acesso em: 15 ago. 2018. 
NEVES, P. M. C.; CERDEIRA, J. P. Memória Organizacional, Gestão do Conhecimento e Comportamentos de Cidadania Organizacional. Perspectivas em Gestão \& Conhecimento, v. 8, n. 1, p. 3-19, 2018. Disponível em: http://dx.doi.org/10.21714/2236-417X2018v8n1p3. Acesso em: 26 abr. 2019.

NONAKA, I. The Knowledge-creating company. Harvard Business Review, v. 69, n. 6, p. 96104, 1991.

NONAKA, I.; TAKEUCHI, H. Criação de conhecimento na empresa: como as empresas japonesas geram a dinâmica da inovação. Rio de Janeiro: Campus, 1997.

NONAKA, I.; TOYAMA, R. The knowledge-creating theory revisited: knowledge creation as a synthesizing process. Knowledge Management Research \& Practice, v. 1, n. 1, p. 2-10, jul. 2003. Disponível em: https://doi.org/10.1057/palgrave.kmrp.8500001. Acesso em: 15 ago. 2018.

NONAKA, I.; TOYAMA, R.; HIRATA, T. Teoria e casos de empresas baseadas no conhecimento: managing flow. Porto Alegre: Bookman, 2011.

OUCHI, W. Teoria Z: como as empresas podem enfrentar o desafio japonês. São Paulo: Nobel, 1985.

PATRIOTTA, G. Organizational knowledge in the making: how firms create, use, and institutionalize knowledge. USA: United States: Oxford University Press, 2003.

PENROSE, E. Teoria do Crescimento da Firma. São Paulo: UNICAMP, 2006.

PEREIRA, D.; CUNHA, S. K. DA; BULGACOV, Y. L. M. Inovação social e aprendizagem organizacional: reflexões sobre sustentabilidade e clusters industriais. In: CONGRESSO DE GESTÃO DE TECNOLOGIA LATINO-IBEROAMERICANO (ALTEC), 15., 2013, Porto. Anais [...]. Porto: 2013. Disponível em: http://www.altec2013.org/programme_pdf/531.pdf. Acesso em: 15 ago. 2018.

PEREIRA, F. C. M.; SILVA, E. F. DA. Criação do Conhecimento Organizacional Baseada nos Capacitadores de Von Krogh, Nonaka e Ichijo: Estudo de Caso. Perspectivas em Gestão \& Conhecimento, v. 8, n. 1, p. 20-43, 2018. Disponível em: http://dx.doi.org/10.21714/2236417X2018v8n1p20. Acesso em: 26 abr. 2019.

POLANYI, M. The tacit dimension. New York: Doubleday \& Co, 1966.

REMOR, L. C. et al. Esquecimento organizacional e suas consequências no processo de aprendizagem organizacional. Revista de Administração Pública, Rio de Janeiro, v. 44, n. 3, p. 667-678, jun. 2010. Disponível em: http://dx.doi.org/10.1590/\$0034-76122010000300006. Acesso em: 15 ago. 2018.

SAMAD, S. The Influence of Innovation and Transformational Leadership on Organizational Performance. Procedia - Social and Behavioral Sciences, v. 57, p. 486-493, out. 2012. Disponível em: https://doi.org/10.1016/j.sbspro.2012.09.1215. Acesso em: 15 ago. 2018. 
SANTANA, R. S. de C.; SOUZA-SILVA, J. C. de. A contribuição do modelo de excelência em gestão pública no processo de aprendizagem organizacional: o caso da Empresa Baiana de Águas e Saneamento. Revista Gestão e Sociedade, v. 6, n. 13, p. 47-68, jan./abr. 2012. Disponível em: https://doi.org/10.21171/ges.v6i13.1563. Acesso em: 15 ago. 2018.

SANZ-VALLE, R. et al. Linking organizational learning with technical innovation and organizational culture. Journal of Knowledge Management, v. 15, n. 6, p. 997-1015, 25 out. 2011. Disponível em: https://doi.org/10.1108/13673271111179334. Acesso em: 15 ago. 2018.

SCORSOLINI-COMIN, F.; INOCENTE, D. F.; MIURA, I. K. Aprendizagem organizacional e gestão do conhecimento: pautas para a gestão de pessoas. Revista Brasileira de Orientação Profissional, São Paulo, v. 12, n. 2, p. 227-240, dez. 2011. Disponível em http://pepsic.bvsalud.org/pdf/rbop/v12n2/10.pdf. Acesso em: 15 ago. 2018.

SENGE, P. A quinta disciplina: arte, teoria e prática da organização de aprendizagem. São Paulo: Best Seller, 1990.

SENGE, P. A quinta disciplina - caderno de campo: estratégias e ferramentas para construir uma organização que aprende. Rio de Janeiro: Qualitymark, 1995.

SHANE, S.; VENKATARAMAN, S. The promise of entrepreneurship as a field of research. Academy of Management Review, v. 25, n. 1, p. 217-226, jan. 2000. Disponível em https://www.jstor.org/stable/259271. Acesso em: 15 ago. 2018.

ŠKERLAVAJ, M.; SONG, J. H.; LEE, Y. Organizational learning culture, innovative culture and innovations in South Korean firms. Expert Systems with Applications, v. 37, n. 9, p. 63906403, 2010. Disponível em: https://doi.org/10.1016/j.eswa.2010.02.080. Acesso em: 15 ago. 2018.

SUTANTO, E. M. The influence of organizational learning capability and organizational creativity on organizational innovation of Universities in East Java, Indonesia. Asia Pacific Management Review, v. 22, n. 3, p.128-135, set. 2017. Disponível em: https://doi.org/10.1016/j.apmrv.2016.11.002. Acesso em: 15 ago. 2018.

SCATOLIN, H. G. A Gestão do Conhecimento nas Organizações: o legado de Nonaka e Takeuchi. Perspectivas em Gestão \& Conhecimento, v. v. 5, n. 2, p. 4-13, 2015. Disponível em: http://www.periodicos.ufpb.br/ojs/index.php/pgc/article/view/21772/14381. Acesso em: 26 abr. 2019.

TEECE, D. J.; PISANO, G.; SHUEN, A. Dynamic capabilities and strategic management. Strategic Management Journal, v. 18, n. 7. p. 509-533, ago. 1997. Disponível em: https://www.jstor.org/stable/3088148. Acesso em: 16 ago. 2018.

TAKAHASHI, A. R. W.; FISCHER, A. L. Descortinando os processos da aprendizagem organizacional no desenvolvimento de competências em instituições de ensino superior. In: ENCONTRO DA ASSOCIAÇÃO NACIONAL DE PÓS-GRADUAÇÃO E PESQUISA EM ADMINISTRAÇÃO, 32., 2008, Rio de Janeiro. Anais [...] Rio de Janeiro: ANPAD, 2008. p. 1-16. Disponível em: http://www.anpad.org.br/admin/pdf/EOR-A1738.pdf. Acesso em: 15 ago. 2018. 
TEECE, D. J. Explicating dynamic capabilities: the nature andmicrofoundations of (sustainable) enterprise performance. Strategic Management Journal, v. 28, n. 13, p. 1319-1350, dez. 2007. Disponível em: https://doi.org/10.1002/smj.640. Acesso em: 15 ago. 2018.

VALDEZ-JUÁREZ, L. E.; LEMA, D. G. de; MALDONADO-GUZMÁN, G. Management of knowledge, innovation and performance in SMEs. Interdisciplinary Journal of Information, Knowledge, and Management, v. 11, p. 141-176, 2016. Disponível em: https://doi.org/10.28945/3455. Acesso em: 15 ago. 2018.

VALIO, R.; GONZALEZ, D.; MARTINS, M. F. Um Estudo Teórico das Características Organizacionais que Influenciam a Gestão do Conhecimento nas Organizações. Perspectivas em Gestão \& Conhecimento, v. 5, n. 2, p. 14-34, 2015.

WANG, C. L.; CHUGH, H. Entrepreneurial learning: past research and future challenges. International Journal of Management Reviews, v. 16, n. 1, p. 24-61, jan. 2014. Disponível em: https://doi.org/10.1111/ijmr.12007. Acesso em: 15 ago. 2018.

WANG, C. L.; AHMED, P. K. Dynamic capabilities: a review and research. International Journal of Management Reviews, Oxford, v. 9, n. 1, p. 31-51, mar. 2007. Disponível em: https://doi.org/10.1111/j.1468-2370.2007.00201.x.

WINTER, S. G. Understanding dynamic capabilities. Strategic Management Journal, v. 24, n. 10, p. 991-995, out. 2003. Disponível em: https://doi.org/10.1002/smj.318. Acesso em: 15 ago. 2018.

ZANGISKI, M. A. S. G.; LIMA, E. P.; COSTA, S. E. G. Aprendizagem organizacional e desenvolvimento de competências: uma síntese a partir da gestão do conhecimento. Produto \& Produção, v. 10, n. 1, p. 54-74, fev. 2009. Disponível em:

https://doi.org/10.22456/1983-8026.7787. Acesso em: 15 ago. 2018.

ZOLLO, M.; WINTER, S. G. Deliberate Learning and the Evolution of Dynamic Capabilities. Organization Science, v. 13, n. 3, p. 339-351, 2002. Disponível em: https://doi.org/10.1287/orsc.13.3.339.2780. Acesso em: 15 ago. 2018.

Artigo recebido em 16/08/2018 e aceito para publicação em 13/07/2019 